\title{
Stenasellus kenyensis n.sp., Crustacea Isopoda Asellota des eaux souterraines du Kénya
}

\author{
par \\ Guy MAGNIEZ* \\ SUMMARY \\ Stenasellus kenyensis n.sp., Crustacea Isopoda Asellota \\ from subterranean waters of Kenya
}

Description of the first cavernicolous Stenasellid discovered in Kenya. St. Kenyensis n.sp. is related to somalian and eurasiatic species of the genus.

Origine du matériel: Le nouveau Sténasellide décrit ci-dessous a été capturé le 2 juin 1974, à 60 pieds de profondeur (Conway Plough Coll.), dans les eaux d'une grotte creusée dans des calcaires, sur la rivière Tiva (Tsavo East National Park, Kenya), entre Mombasa et Nairobi, assez largement au Sud de l'équateur.

L'holotype de l'espèce, dont les appendices sont représentés plus loin, est un ot de $16,1 \mathrm{~mm}$ de long. Deux paratypes ơ de 15 et $17 \mathrm{~mm}$ m'ont ensuite été communiqués. La + n’a pas été observée.

Je remercie très sincèrement Monsieur le Docteur $\mathrm{A}$. J. Bruce, de Mombasa, ainsi que Monsieur le Professeur Th. Monod, Membre de l'Académie des Sciences de France, de la confiance qu'ils m'ont témoignée en me demandant de décrire Stenasellus kenyensis n.sp.

Aspect général: Assez semblable à St. costai Lanza, Chelazzi et Messana, 1970, la forme géante $(26 \mathrm{~mm})$ des eaux souterraines de Somalie, mais avec une phanérotaxie plus réduite. Corps robuste, environ 5 fois plus long que large. Tégument épais et translucide. Coloration rouge sur le vivant ("brilliant scarlet", selon A. J. Bruce, in litt.), mais jaunâtre, avec des condensations brunâtres dans les tissus, due à la fixation et à l'altération post-mortem des pigments hémolymphatiques, comme cela se produit chez les Stenasellides mis en alcool. Capsule céphalique globuleuse (fig.6), plus large ( $2,4 \mathrm{~mm}$ environ) que longue ( $1,8 \mathrm{~mm}$ environ), à marge rostrale régulièrement concave et marges latérales convexes, peu mobile par rapport au péréionite I. Une petite tache brunâtre paire est visible par transparence dans les tissus du céphalon, près des angles rostraux, au dessus de l'insertion des antennes (cf. fig. 6). De telles formations ont été repérées en premier lieu par Lanza, Chelazzi et Messana sur St. costai et ils ont pensé y voir des vestiges oculaires.

\footnotetext{
* Laboratoire de Biologie animale et générale, Université de Dijon, 6, Bd. Gabriel, 21000 Dijon, France.
} 


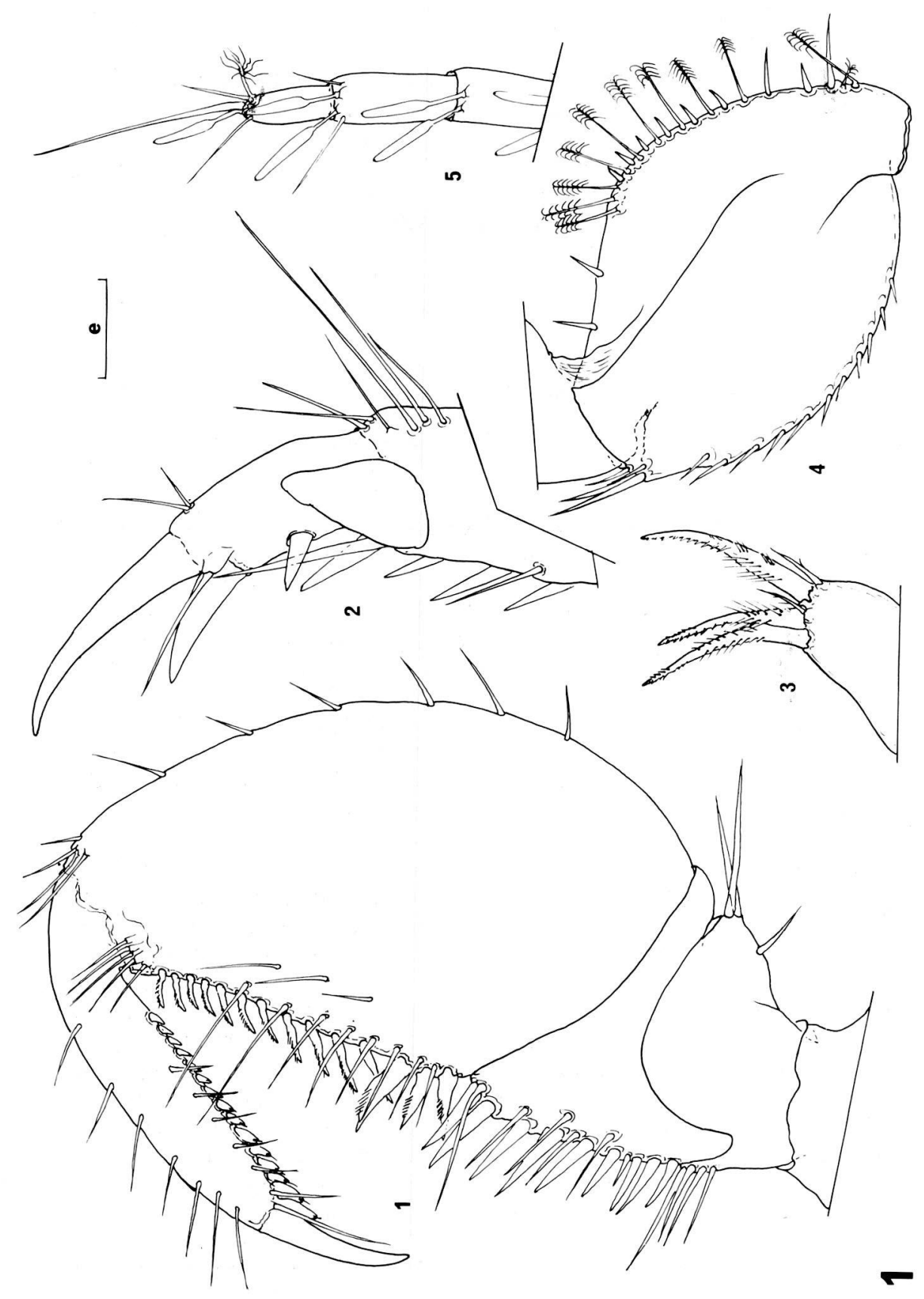


Portions pleurales des péréionites bien développées, masquant presque complètement les coxopodites II-IV en vue dorsale, mais laissant apparaitre, au niveau d'une échancrure latéro-postérieure, la marge externe des coxopodites V-VII (fig. 14-15). Largeur du corps passant de 3,00 mm (péréionite I) à 3,32-3,36 mm (péréionites III et IV) et revenant à 3,24 $\mathrm{mm}$ (péréionite VII). Forte armature marginale de soies, surtout aux angles rostraux du péréionite I et aux angles caudaux des tergites de tous les péréionites, en particulier les postérieurs. Pléonites libres I et II très développés (fig. 14-15), ensemble aussi longs $(1,84 \mathrm{~mm})$ que le péréionite VII $(1,80$ $\mathrm{mm})$, plus larges $(3,01 \mathrm{~mm})$ que le pléotelson $(2,92 \mathrm{~mm})$. Ce dernier plus long $(4,20$ $\mathrm{mm}$ ) que large et à pointe caudale arrondie (fig. 14).

Appendices céphaliques: Antennules d'environ $3 \mathrm{~mm}$. Fouet de 16-17 articles dont les 6 proximaux sans lames olfactives; les 9 suivants avec 2 L.O. chacun (longueur 100-110 $\mu$ ); l'avant-dernier avec 1 seule L.O. (fig.5). Antennes de 9,8 mm environ, atteignant la marge antérieure du péréionite $\mathrm{V}$; squama bien développée, sur le 3 ème article de la hampe (fig. 13) et portant 2 fortes tiges lisses; fouet de 70-75 articles. Mandibule typique de Stenasellidae, très indurée; lacinie avec 4 fortes dents; lacinie mobile avec également 4 dents ( $\mathrm{mdb}$. gauche); lobe mandibulaire avec une douzaine de tiges falciformes denticulées; lobe molaire avec une rangée continue de soies marginales longues et souples; palpe triarticulé normal. Lobe externe de la maxillule avec de fines sétules sur la marge interne; 12 fortes épines denticulées distalement et 6 tiges bipectinées sur la marge distale; lobe interne avec 3 fortes tiges à ramifications polymorphes et 2 courtes soies simples distales (fig. 3 ). Lobes externe et moyen de la maxille avec une rangée distale de 14-15 lames falciformes monopectinées, décroissant vers l'intérieur; lobe interne avec une trentaine de fortes tiges plumeuses ou dentelées polymorphes, placées sur deux rangs et de fines sétules sur la marge interne.

Maxillipèdes: Typiques de Stenasellidae (cf. Magniez 1974, p.11), avec endite à 4 crochets rétinaculaires.

Péréiopodes: Relativement courts et très robustes (indice d'un comportement fouisseur très développé? ). Longueurs absolues: 4,31-4,64 - 4,79-4,75 - 5,21-6,27 et $7,36 \mathrm{~mm}$. Longueur rapportée au péréiopode II: 0,93-1,00 - 1,03-1,02 - 1,12-1,35 et 1,59 .

Péréiopodes I préhensiles fortement armés (fig. 1). Marge sternale du carpopodite avec nombreuses tiges simples. Marge sternale du propodite avec 3 tiges ensifor-

PLANCHE 1.

St. kenyensis n.sp.; ơ de $16,1 \mathrm{~mm}$ :

1. Péréiopode I gauche, face sternale; e $=100 \mu$.

2. Péréiopode II gauche, face caudale; $\mathrm{e}=100 \mu$.

3. Lame interne de la maxillule gauche; $\mathrm{e}=100 \mu$.

4. Basipodite du péréiopode VI gauche, face caudale; $\mathrm{e}=333 \mu$.

5. Extrémité du fouet de l'antennule gauche; $\mathrm{e}=100 \mu$. 

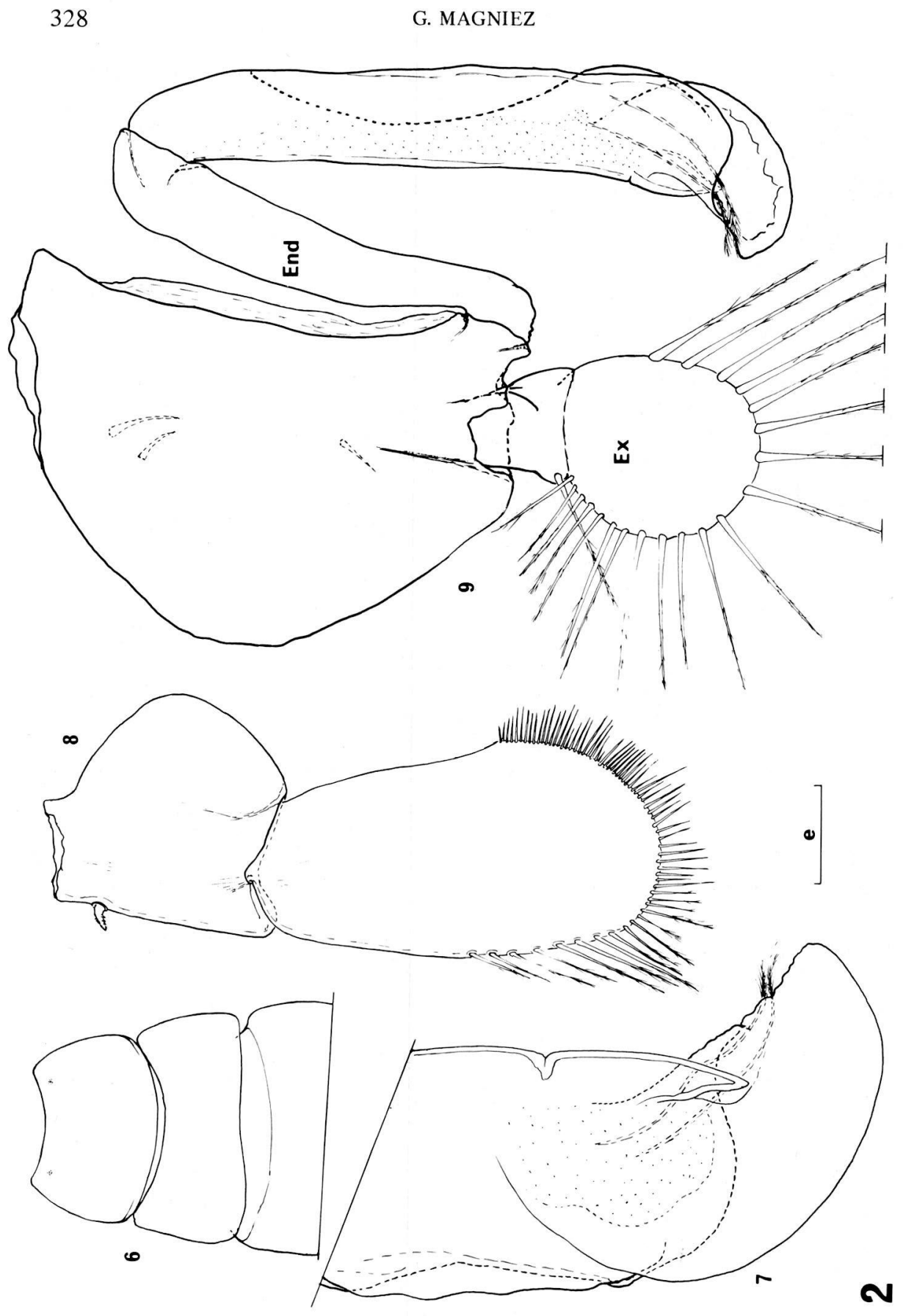
mes épaisses dressées et 11 tiges denticulées récurrentes. Dactylopodite avec une rangée subunguéale continue de robustes lames obliques.

Basipodites des péréiopodes II-VII avec une carène tergale très marquée et de nombreuses soies sensorielles "en palmier". Dactylopodite avec 2 épines sternales, la proximale petite, la distale très longue et robuste, insérée juste sous l'ongle (fig. 2).

Papilles génitales insérées très latéralement, à l'angle interne des coxopodites des péréiopodes VII (segment thoracique 8 ); très longues $(1350 \mu)$ et grêles (diamètre $160 \mu)$, mais relativement rigides et à extrémité biseautée.

Pléopodes I o: Au repos, ils sont plaqués sur la face sternale des pléopodes II et les protègent complètement. Protopodite subpentagonal avec rétinacle formé d'un seul crochet très basal. Exopodite 2 fois plus long que le protopodite, avec une rangée continue d'environ 70 soies dont une quinzaine plus longues (jusqu'à $430 \mu$ ) et plumeuses, sur la marge distale-interne; les autres plus courtes $(100-250 \mu)$, lisses et très serrées (fig. 8).

Pléopodes II o: (fig. 9) protopodite plus long $(1100 \mu)$ que large $(800 \mu)$, à tégument induré. Face tergale glabre; face sternale avec seulement 3 courtes épines lisses. Exopodite court $(550 \mu)$ avec article proximal muni d'une seule soie plumeuse sur la marge externe; article distal subcirculaire, à face tergale concave, avec une vingtaine de longues (200-500 $\mu$ ) soies plumeuses périphériques. Endopodite géniculé au repos (l'article proximal se disposant contre la face interne, plane, du protopodite). Article proximal long $(800 \mu)$ et grêle, rigide et bien articulé avec le protopodite. Article distal très long $(1300 \mu)$ et grêle, aplati dorsoventralement, mais rigide et terminé par un éperon induré, comme chez St. costai (cf. Lanza, Chelazzi et Messana 1970, fig. 28, p.151). Structure générale caractéristique du genre Stenasellus s.str. Infundibulum proximal afférent très oblique, sternal. Orifice efférent terminal très large, tergal, délimité par une lèvre tergale réduite et une lèvre sternale hypertrophiée (fig. 7,9) se prolongeant en direction externe. Une double protubérance axiale sétigère fait saillie à l'angle externe de l'orifice et prolonge deux renforcements chitineux réfringents internes de l'organe copulateur. L'aspect général de l'appendice rappelle celui de $S t$. costai et de $S t$. pardii, mais l'extrémité de l'endopodite est très différente, ce qui permet de considérer St. kenyensis comme une espèce totalement indépendante des deux formes somaliennes, bien qu'appartenant à la même lignée évolutive du genre Stenasellus s. str. .

\section{PLANCHE 2.}

St. kenyensis n.sp.; ô de $16,1 \mathrm{~mm}$ :

6. Céphalon et péréionites antérieurs en vue dorsale; les taches pigmentaires intracéphaliques ont été indiquées; $\mathrm{e}=1280 \mu$.

7. Extrémité du second article de l'endopodite du pléopode II, face sternale, montrant l'éperon terminal induré et la lèvre sternale de l'orifice efférent; $\mathrm{e}=100 \mu$.

8. Pléopode I gauche, face sternale; $\mathrm{e}=333 \mu$.

9. Pléopode II gauche, face tergale: ex. = exopodite; end. = endopodite $; \mathrm{e}=200 \mu$. 


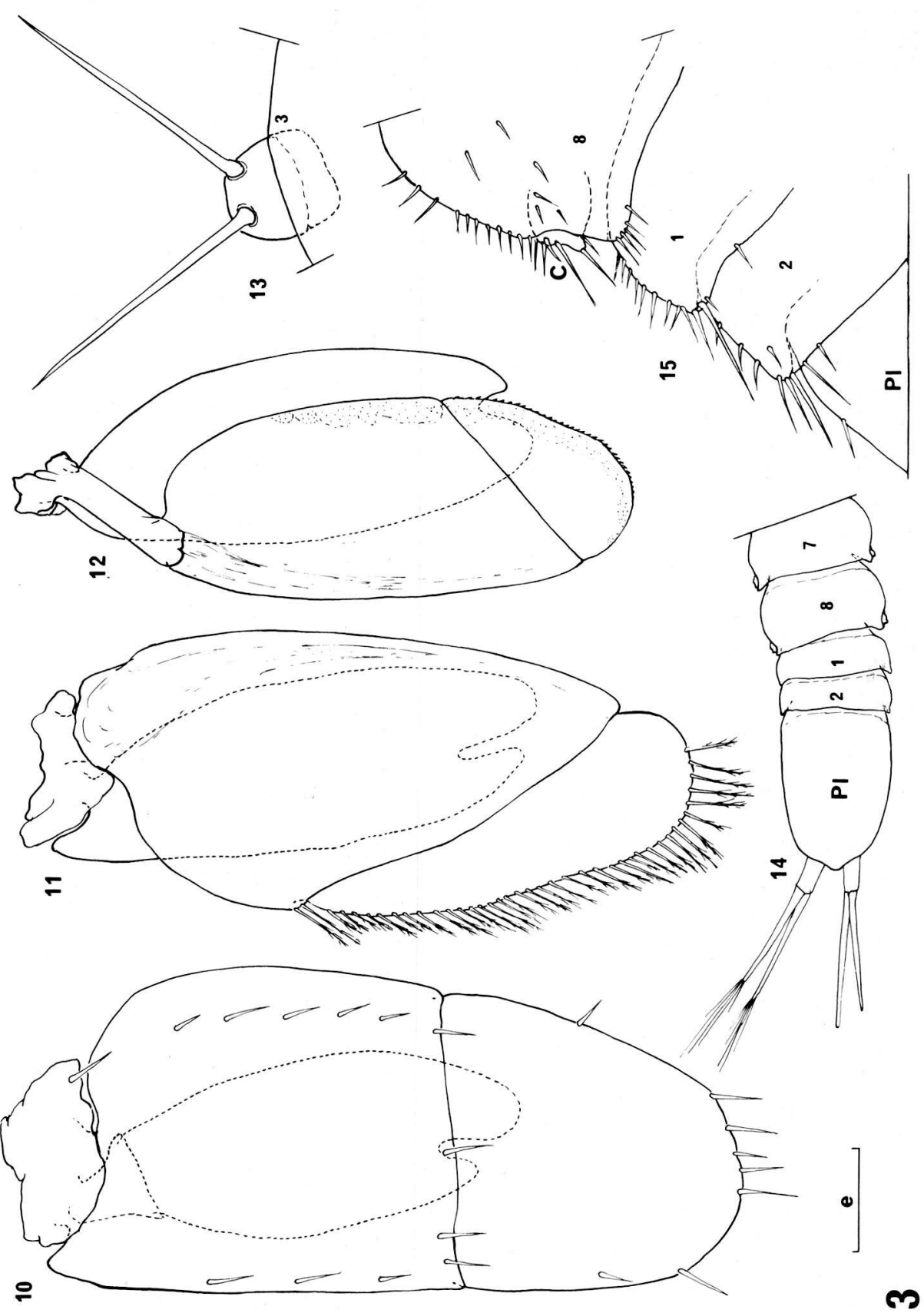


Pléopodes III, IV et V: Endopodites ovalaires, charnus et respiratoires, plus petits que leur exopodite respectif. Tous possèdent une échancrure de la marge distale (fig. 10,11,12) que l'on connaît également chez la forme Stenasellus sp.découverte par Cl. Boutin (1971) dans des grottes du Cambodge (cf. Magniez 1974, p. 42). Chez St. costai, les endopodites sont entiers, tandis que chez la petite espèce $S t$. pardii, ils sont entièrement bilobés (cf. Lanza 1966, fig. 24, 25, 27, p. 232). Exopodite III (opercule) induré et très allongé (fig. 10). Exopodite IV (fig. 11) ovalaire, induré et lamellaire, avec suture interarticulaire très oblique. Marge interne et distale portant près de 40 fines soies un peu plumeuses. Exopodite $V$ (fig. 12) régulièrement ovalaire, induré et lamelleux, à suture interarticulaire oblique. Une aire glandulaire sur les marges interne et distale est couverte d'écailles cuticulaires auriculées.

Uropodes: A peu près aussi longs que le pléotelson (fig. 14), avec endopodite légèrement plus long que l'exopodite; les deux rames avec un long pinceau de soies à direction caudale.

Affinités: La nouvelle espèce est un Sténasellide typique. Elle appartient sans ambiguité au genre Stenasellus s.str. (cf. Magniez 1974, p. 24 et suiv.), c'est-à-dire qu'elle est, comme les formes somaliennes, apparentée aux espèces eurasiatiques du groupe, mais non aux espèces d'Afrique occidentale et du bassin du Congo, qui ont été classées dans des genres différents (Lanza 1966, Magniez 1966, 1974). La répartition actuellement connue des espèces du genre Stenasellus (péninsule ibérique, Midi de la France, Corse, Sardaigne, Toscane, péninsule balkanique, Turkménistan, Cambodge et Afrique orientale) semble liée à des rivages anciens (Lutétiens?) de la Mésogée et de ses annexes épicontinentales (bras de mer russes, canal de Mozambique). Si tel est le cas, il devrait être possible d'en découvrir de nouvelles formes du Proche-Orient à l'Inde.

St. kenyensis n.sp., bien qu'apparenté aux formes somaliennes, s'en distingue facilement, par des caractères spécifiques nets, portant en particulier sur l'ensemble des pléopodes I à $\mathrm{V}$ et aucune confusion n'est possible entre ces trois espèces. Il permet de vérifier, une fois de plus, que si l'organe copulateur possède la même architecture d'ensemble dans le groupe, sa partie terminale diffère toujours, dans ses détails, d'une espèce à l'autre.

\section{PLANCHE 3.}

St. kenyensis $\mathrm{n} . \mathrm{sp}$; ${ }^{\dagger}$ de $16,1 \mathrm{~mm}$ :

10. Pléopode III gauche, face sternale; endopodite indiqué en pointillés; $\mathrm{e}=500 \mu$

11. Pléopode IV gauche, face sternale; endopodite partiellement en pointillés; $\mathrm{e}=500 \mu$.

12. Pléopode V droit, face sternale; endopodite partiellement en pointillés; $\mathrm{e}=500 \mu$.

13. Ecaille (squama) de l'antenne, portée par le 3ème article de la hampe (3); $\mathrm{e}=100 \mu$.

14. Région postérieure du type, face tergale: 7 et $8=$ derniers segments thoraciques; 1 et $2=$ pléonites libres I et II; PI. = pléotelson. Les uropodes ont été schématisés; e $=2666 \mu$.

15. Portion latérale gauche, plus grossie $(e=640 \mu)$ du segment thoracique 8 et des pléonites 1 et $2 ; \mathrm{C}=$ coxopodite du péréiopode VII. 
La découverte d'un Stenasellus s.str. au Kénya est d'un grand intérêt biogéographique, puisque la nouvelle espèce vit à quelques $900 \mathrm{~km}$ au S-SW des localités somaliennes de St. pardii. On peut se demander si d'autres formes de la lignée ne se sont pas installées dans les eaux souterraines de régions encore plus méridionales de l'Afrique orientale.

\section{RESUME}

Description du premier Stenasellus cavernicole découvert au Kénya. St. kenyensis n.sp. est apparenté aux espèces somaliennes et aux formes eurasiatiques du genre.

\section{BIBLIOGRAPHIE}

BIRSTEIN, J. A. \& STAROSTIN, I. V. 1949 - Sur une forme nouvelle pour l'URSS de Stenasellus, provenant du Turkménistan (en russe). C.R.Acad. Sc. Moscou.N.S., 69, 691-694.

BOUTIN, Cl. 1971 - Observations biospéologiques en Asie du Sud-Est. Ann. Fac.Sc. PhomPhen, 4, 168-186.

LANZA, B. 1966 - Stenasellus pardii sp.n. della Somalia e note sistematische sugli Stenasellinae (Crustacea Isopoda). Monit.Zool. Ital., 74, 221-256.

LANZA, B., CHELAZZI, L. \& MESSANA, G. 1970 - Stenasellus costai sp.n., Isopode freatobio gigante della Somalia. Monit. Zool. Ital. N.S., III, 5, 133-158.

MAGNiEZ, G. 1974. Données faunistiques et écologiques sur les Stenasellidae (Crustacea Isopoda Asellota des eaux souterraines). Int. J. Speleol. 6, 1, 1-80.

MONOD, Th. 1924 - Sur quelques Asellides nouveaux des eaux douces de l'Afrique du Nord. Bull. Soc. Hist. Nat. Afr. Nord, 15, 7, 327-336. 